from van't Hoff's study of osmotic pressure, and his extension of the gas laws to solutions. This remarkable work was followed, at a short interval, by Arrhenius's hypothesis of electrolytic dissociation, a conception that has left its mark deep on the physico-chemical research records of the past thirty years. This hypothesis has been the guiding principle in countless investigations, and although it presents difficulties not yet satisfactorily solved, and appears to require modification in some respects, notably in regard to the rôle of hydration, it holds the ground to-day as the most acceptable and intelligible interpretation of the properties of electrolyte solutions. The history of the electrolytic dissociation theory may be fairly described in Larmor's words: "In the case of every successful scientific theory the time must come when its first easy triumphs become exhausted, and what prominently confronts the investigator are its outstanding defects and diffculties." Such is the present position in regard to the ionisation theory, and during recent years there has been a concentration of effort on such outstanding problems as the application of the mass action law to strong electrolytes, the catalytic action of ions, and the differences existing between the values of the ionisation ratio deduced for one and the same electrolyte by the osmotic and conductivity methods respectively.

The decade in which the theories of van't Hoff and Arrhenius were propounded saw also the establishment of the first journal exclusively devoted to the record of physico-chemical research. The first number of the Zeitschrift für physikalische Chemie appeared in 1887 , and an inspection of the early volumes reveals the extraordinary variety and attractiveness of the problems that were being attacked under the ægis of the new science, and on the more definitely quantitative lines for which this branch of chemistry stands. It was not long before the influence of physical chemistry began to be apparent beyond its own borders in a renascence of inorganic chemistry which continues to the present day. Important reactions between well-known substances, regarded as completely worked out, have been explored afresh in the light of physico-chemical principles, and have yielded an extraordinary amount of valuable quantitative data. In this connection one might refer to the phase rule and its practical utility in connection with the conditions of existence of salt hydrates, the constitution of alloys, and various technical problems.

Prominent amorig the later developments of physical chemistry has been the examination of matter in a condition coarser than that corresponding with the molecular state. The study of mechanical suspensions, and the investigation of colloidal solutions with the aid of the ultramicroscope, have opened up a whole new world of fascinating phenomena, and bridged the gap between the visible particle and the molecule. Perrin's eposh-making count of the particles at different levels in a vertical column of a mechanical suspension, and the evaluation of the Avogadro constant which follows therefrom, have notably extended the validity of the gas laws, and supplied at the same time definite quantitative proof of the molecular movements postulated by the kinetic gas theory. Of extraordinary interest also in this connection is the fact that purely physical evidence, based on the atomic character of electricity and depending on measurements of the elementary electric charge, gives strong support to the Avogadro conception.

At the present moment fresh means of attacking the still unsolved problems of the physicochemical field are being developed. Planck's quantum theory, for example, coupled with such experimental work as that on the heat capacity of solids at low temperatures, and on the origin and relationship of spectral lines, appears likely to have a notable influence on the future of physical chemistry. The thorough investigation of colloids along physico-chemical lines, which is actively proceeding to-day, promises to throw light on many problems which are of interest not only from the purely scientific point of view, but also to the industrial chemist. The sister sciences, too, are vitally concerned in the exploitation of this field, and, indeed, the physical chemist of to-day may point with legitimate pride to the fact that the principles of his science are welcomed by the metallurgist, the physiologist, the geologist, and others, as valuable aids in the elucidation of their respective problems. This everwidening influence is the guarantee of the future vitality of physical chemistry.

\title{
THE INFLUENCE OF INVESTIGATIONS ON THE ELECTRICAL PROPERTIES OF GASES ON OUR CONCEPTIONS OF THE STRUCTURE OF MATTER.
}

\author{
By Sir J. J. Thomson, O.M., Pres. R.S.
}

\begin{abstract}
A LL workers in science owe much to Nature, and so I am glad to comply with the request of its Editor to write a few words on the progress of some branch of physics in the fifty years since NATURE was started. I shall confine myself to the effect which results obtained by investigations NO. $26 \mathrm{IO}$, VOL. IO4]
\end{abstract}

on the electrical properties of gases have had on our conceptions of the structure of matter and the potentiality of further applications of these results to increase our knowledge of physical and chemical problems. In these investigations we study atoms and molecules when they are charged with electri- 
city, and the success which has been obtained is due in the main to the fact that the methods by which we can detect the existence and follow the behaviour of these charged particles are almost infinitely more powerful than those which are available when the particles are uncharged. We can by the aid of their charges detect the presence of a few thousand atoms, while the most delicate methods of chemical analysis will scarcely detect a million million. Again, when an atom or molecule is charged we can by acting upon it by electrical forces increase its energy a million-fold, and thus enable it to produce effects by which its presence can be detected. We obtain in this way very powertul and accurate methods for measuring some of the fundamental constants associated with atoms and molecules. We know now, for example, with great precision the masses of the molecules of the different gases; we owe this to the study of their electrical properties.

Again, the study of the positive rays has shown that all the atoms of an element have to a very high degree of approximation the same mass, and has disposed of the idea that the atomic weight only represented an average value taken over a considerable range. The positive rays, too, have demonstrated the existence in most gases of both atoms and molecules; not only have they shown that atoms exist, they have also proved the independent existence of the radicles of organic chemistry such as $\mathrm{CH}, \mathrm{CH}_{2}, \mathrm{CH}_{3}$. These rays will, I think, in the future play a considerable part in the determination of the atomic weight of those elements which can exist in the gaseous form, as they furnish a method which is independent of impurities, and can distinguish between "isotopes," should such exist. The rays provide a powerful method for detecting new elements and compounds, as they demand only an infinitesimal amount of material and the atomic weight of the new body can be calculated at once from the position of its line in the positive ray spectrum. As a side issue the rays show the complexity of the conditions when electricity passes through compound gases. $I$ have found cases in which there were as many as thirty-seven different-types of positive carriers at work simultaneously.

The convection of negative electricity presents a remarkable contrast, for one of the most striking results of the study of the electrical properties of gases is that at very low pressures the carriers of negative electricity are not atoms or molecules, but electrons, the mass of which is only about I I I oo of that of the smallest known atom, that of hydrogen; these carriers are unaltered in character whatever changes may take place in the nature of the gas through which the electricity is passing. These electrons can be obtained from atoms of every kind, so that they form an integral part of the normal atom. The number of electrons in an atom which are not fixed too rigidly to be shaken when struck by Röntgen rays has been determined, and it has been found that the number No. 26 IO, VOL. IO4] of such electrons in an atom of any element is equal to the atomic number of the element. The positive rays show that the atoms of elements other than hydrogen which occur in these rays must contain more than one electron, for atoms which have lost two or more electrons are a common feature in these rays; mercury atoms have been observed which have lost as many as eight electrons. The speed which the electrons may attain is very great; some of the electrons emitted by radio-active substances (the $\beta$-rays) travel at a speed only a few per cent. less than that of light.

The source of the mass of the electrons is interesting; it was known before they were discovered that a charged body had in virtue of its charge a larger mass than an uncharged one, the difference increasing as the size of the body diminished. The result at that time looked very academic, as even molecules were far too large for the effect to be appreciable; the result became of practical importance when electrons (the linear dimensions of which are only about one-hundredthousandth part of those of atoms) were discovered; and the experiments indicate that the whole of the mass of an electron is due to its charge. Mass of this kind depends upon the velocity and becomes infinite when the velocity is that of light. The mass of the electrons accounts, however, for only a minute fraction of that of the atom of which they form a part.

Since we know the number of electrons in an atom, the problem of finding the structure of the atom is that of finding the configuration of these electrons when they are in equilibrium under their mutual repulsions and whatever forces may be exerted upon them by the positive charges. The solution of this problem would give representations of the structure of the atoms of the various elements. The consideration of the positions of equilibrium when two such atoms of the same or different kinds are brought near together would lead to clear views as to what constitutes chemical combination and the conditions under which it is possible. This is one of the problems which call most urgently for solution. It must be noticed, however, that we cannot explain the properties of the atoms of the elements by a system of positive and negative point charges exciting forces varying inversely as the square of the distance. These would not give rise to systems of atoms sharply limited to definite and distinct types, but to systems passing continuously from one type to another. To get the requisite definiteness in the model atom we must introduce some other condition, such, for example, as that the force between the positive and negative forces is not always an attraction varying inversely as the square of the distance, but that it changes from attraction to repulsion at definite distances (such distances giving a length to measure the size of the atom), or we may assume some condition such as is imposed by the quantum theory, which rules out all but a small fraction of the solutions otherwise possible. 